\title{
Redescubrimiento de Tayassu pecari (Artiodactyla: Tayassuidae) en el Refugio Nacional de Vida Silvestre Mixto Caño Negro, Costa Rica
}

\author{
Sol Guerrero Ortiz \& Francisco José Morazán Fernández \\ Instituto Internacional en Conservación y Manejo de Vida Silvestre, Universidad Nacional de Costa Rica. Apartado 1350-3000, Heredia, Costa \\ Rica; solgo.guerrero@gmail.com, cyclopes21@gmail.com
}

Recibido 07-VI-2016 • Corregido 27-VI-2016 • Aceptado 24-VII-2016

\begin{abstract}
Rediscovery of Tayassu pecari (Artiodactyla: Tayassuidae) in the Refugio Nacional de Vida Silvestre Mixto Caño Negro, Costa Rica. The white lipped peccary (Tayassu pecari) is a widely distributed Neotropical species that can be found from northern Argentina to southern Mexico. According to the IUCN, the white lipped peccary is vulnerable throughout its distribution range. In Costa Rica their distribution has decreased in $89 \%$, its populations are in danger of extinction and are restricted to a few protected areas. This document presents the details of the first records of the presence of white lipped peccary in the Mixed National Wildlife Refuge of Caño Negro, where it was previously considered to be locally extinct. During 2013, 52 in depth interviews were conducted in several communities near the refuge. Between September and December 2015, eight camera traps were placed in farms close or within the refuge. In ten of the conducted interviews, people confirmed that there is a small population of these animals. Most people believe they live in a high and secluded sector of the refuge known as "Terrón". Of the eight camera traps that were used, two were placed in this sector. One of these cameras registered a pack of white lipped peccary composed of at least eight individuals including adults and juveniles. Local ecological knowledge serves as a complement for technical knowledge and as a guide for planning and focusing efforts during the development of ecological research, such is the case of the present study. The confirmation of the presence of white lipped peccary is a finding of great importance since it can serve to increase the conservation measures that are applied in the refuge.
\end{abstract}

Key words: Arenal North Huetar Conservation Area, camera trap, local ecological knowledge, tropical wet forest, white-lipped peccary.
RESUMEN: El chancho de monte (Tayassu pecari), es una especie Neotropical de amplia distribución que se encuentra desde el norte de Argentina hasta el sur de México. De acuerdo con la UICN, esta especie se considera en estado vulnerable en todo su rango de distribución. En Costa Rica su distribución ha disminuido en $89 \%$ y se encuentra restringida a pocas áreas protegidas, lo que la coloca en la lista de especies en peligro de extinción. En este documento presentamos los detalles de los primeros registros de la presencia de chancho de monte dentro del Refugio Nacional de Vida Silvestre Mixto Caño Negro, donde anteriormente se consideraba localmente extinto. En el 2013, realizamos 52 entrevistas a profundidad en diversas comunidades cercanas al refugio. Entre septiembre y diciembre del 2015, colocamos ocho cámaras trampa en fincas aledañas o dentro del refugio. En diez de las entrevistas realizadas, los pobladores informaron sobre la existencia de una población pequeña de estos animales. La mayoría creen que se encuentran en un sector alejado y elevado del refugio conocido como "Terrón". De las ocho cámaras utilizadas, dos de ellas se colocaron en fincas correspondientes a dicho sector. En una de estas cámaras se registró una manada de chachos de monte de al menos ocho individuos, compuesta tanto por adultos como juveniles. El conocimiento ecológico local sirve como complemento del conocimiento técnico y como guía para planificar y focalizar esfuerzos durante el desarrollo de investigaciones ecológicas, como en el caso del presente trabajo. Confirmar la presencia del chancho de monte es un hallazgo que puede servir para aumentar las medidas de conservación que se aplican en el refugio.

Palabras clave: Área de Conservación Arenal Huetar Norte, bosque húmedo tropical, cámara trampa, chancho de monte, conocimiento ecológico local, pecarí de labios blancos.
El pecarí de labios blancos, conocido en Costa Rica como chancho de monte (Tayassu pecari, Link 1795), es un ungulado de tamaño medio. Presenta una longitud entre $75 \mathrm{~cm}$ y $1 \mathrm{~m}$, una altura al pecho entre 44 y 57,5 $\mathrm{cm}$, y un peso entre 25 y $42 \mathrm{~kg}$. El pelaje es áspero y la coloración va de café oscuro a negro, con áreas blancas en la región pélvica y en el lado dorsal del cuello (Mayer
\& Wetzel, 1987).Es un animal terrestre que presenta hábitos tanto diurnos como nocturnos y vive en manadas que pueden estar conformadas de 50 a 300 individuos (Wainwrigth, 2007). Es una especie Neotropical de amplia distribución abarcando zonas de bosques húmedos, sabanas y bosques secos, desde el norte de Argentina hasta el sur de México (Sowls, 1997). La pérdida de su 
hábitat y la cacería indiscriminada son los principales problemas que enfrenta esta especie (Carrillo et al., 2002; Leonardi et al., 2010; Briceño-Méndez et al., 2014).

El chancho de monte se considera vulnerable en todo su rango de distribución, de acuerdo con la UICN (Keuroghlian et al., 2013). Sin embargo, se encuentra en peligro de extinción en México y Costa Rica, ha desaparecido en El Salvador y se encuentra en bajas densidades poblacionales en el resto de Centroamérica (Carrillo et al., 2002; Leonardi et al., 2010; Briceño-Méndez et al., 2014). En el pasado en Costa Rica se distribuía en hábitats disponibles en todo el país. Sin embargo, su distribución ha disminuido en un $89 \%$ en comparación con su distribución histórica (Altrichter et al., 2012). Actualmente sus poblaciones se encuentran restringidas solo a las siguientes áreas protegidas: Parque Nacional Tortuguero, Parque Internacional La Amistad (Carrillo et al., 2002); se ha reportado en las partes altas del Sector San Cristóbal del Parque Nacional Guanacaste (Alfaro, 2006; Amit, 2006), Parque Nacional Rincón de la Vieja (Leonardi et al., 2010; Amit \& Fernández, 2012), y en el Parque Nacional Santa Rosa no se han registrado pero se han identificado restos de chancho de monte a partir de la excreta de un jaguar (Montalvo, 2012). Finalmente, según estudios realizado por Chinchilla (1997), en el Parque Nacional Corcovado se encontró que el chancho de monte es una de las presas principales para el jaguar.

Como parte de dos investigaciones del Instituto Internacional en Conservación y Manejo de Vida Silvestre (ICOMVIS) de la Universidad Nacional de Costa Rica (UNA), referentes al conflicto entre ganaderos y grandes felinos, se realizaron entrevistas a los pobladores y se colocaron cámaras trampa. Este documento presenta los detalles del primer registro de la presencia de chancho de monte dentro del Refugio Nacional de Vida Silvestre Mixto Caño Negro (RNVSM-CN), área natural protegida que no está actualmente considerada como parte de su área de distribución.

\section{MATERIALES Y MÉTODOS}

Área de estudio: El RNVSM-CN es un humedal continental conformado por un sistema lagunar generado por el desbordamiento del Río Frío. Pertenece al Área de Conservación Arenal Huetar Norte, al noroeste de Costa Rica (Escalera-Reyes et al., 2013). Tiene una extensión de 9969 ha y corresponde a la ubicación geográfica $10^{\circ} 54^{\prime}$ - $10^{\circ} 47^{\prime} \mathrm{N}$ y $84^{\circ} 42^{\prime}-84^{\circ} 47^{\prime} \mathrm{W}$ (Coloma et al., 2009). La precipitación anual es entre 2500 y $3500 \mathrm{~mm}$, la temperatura oscila entre 24 y $26^{\circ} \mathrm{C}$ y la humedad relativa promedio es del $80 \%$ (Cabrera et al., 2003). Se clasifica como refugio de propiedad mixta porque está compuesto tanto por terrenos del Estado (40\%), como por propiedad privada (60\%) (Coloma et al., 2009). La presencia permanente o estacional de las aguas tiende a condicionar los tipos de asociaciones vegetales que se desarrollan. El refugio se caracteriza por tener bosques húmedos tropicales (Holdridge, 1979). Las áreas poco drenadas están dominadas por la palma Raphia taedigera (yolillo) (Coloma et al., 2009), pero también hay presencia de pastizales.

Obtención de datos: En el 2013, como parte del proyecto "Estado del conocimiento del jaguar y puma en las comunidades de la zona norte de Costa Rica y su relación con el miedo, los mitos y el conflicto entre los seres humanos", realizamos en diversas comunidades cercanas al RNVSM-CN, 52 entrevistas a profundidad con el uso de una guía de entrevista que contenía los temas y subtemas por cubrir, de acuerdo a los objetivos informativos de la investigación sobre conocimiento ecológico local de fauna silvestre.

Entre septiembre y diciembre del 2015, como parte del proyecto "Conflicto humano-felinos mayores y propuesta de acciones para su mitigación y prevención, en el Refugio Nacional de Vida Silvestre Mixto Caño Negro, Costa Rica", colocamos ocho cámaras trampa (ScoutGuard modelo SG570B), en fincas aledañas o fincas dentro del RNVSM-CN que tuvieran presencia de alguno de los principales tipos de cobertura vegetal. Seleccionamos puntos favorecidos y rutas transitadas (basadas en el conocimiento ecológico local), donde anteriormente los dueños habían observado rastros de fauna como en senderos, brechas o cerca de cuerpos de agua (Medellín et al., 2006; Chávez et al., 2013). Cada cámara permaneció activa 60 días consecutivos con revisiones periódicas cada diez días.

\section{RESULTADOS}

En diez de las entrevistas $(\mathrm{N}=52)$ se reportó la presencia tanto histórica como actual, de manadas de chanchos de monte. Cinco informantes indicaron que existe una población pequeña de estos animales en un sector alejado y elevado del refugio conocido como "Terrón". Según expresaron las personas entrevistadas y los funcionarios del SINAC, esta es una de las zonas más conservadas dentro del refugio, pero donde se practica más cacería. Adicionalmente se reportaron chanchos de monte en otros lugares circundantes a la zona como "Nueva Esperanza" (tres reportes), "San Antonio" (un reporte) y "La Unión" (un reporte). 


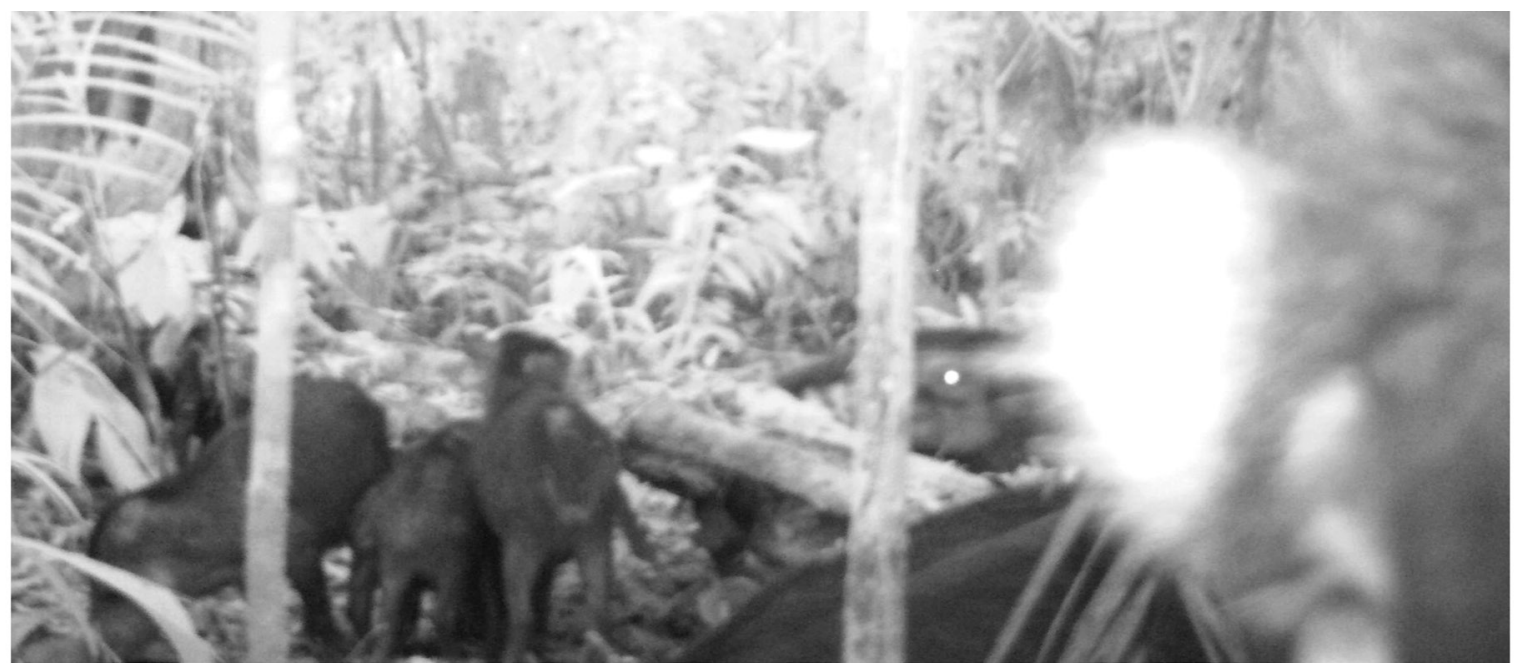

Fig. 1. Imagen de chancho de monte capturada en noviembre del 2015 con cámara trampa en bosque húmedo tropical, dentro del Refugio Nacional de Vida Silvestre Mixto Caño Negro, Costa Rica.

En un avistamiento se reportó una manada de entre 50 y 100 individuos, aunque los informantes aseguran que antes había una cantidad mucho mayor a la actual. Todos los informantes que reportan presencia de chanchos de monte, describieron muy bien las características físicas de estos animales y no los confunden con los saínos (Dicotyles crassus) aunque también están presentes en el refugio (Wainwrigth, 2007). Reportaron que los chanchos además de ser más grandes, son muy agresivos, negros, con colmillos largos y un olor muy fuerte. Principalmente se ha observado a estos animales cerca de distintos tipos de palmas de cuyos frutos se sabe que se alimentan (Bodmer et al., 1997; Beck, 2006). Todos los informantes son originarios de esta zona (diez) y la mayoría (nueve) eran adultos de la tercera edad. Cabe destacar que algunos fueron cazadores y están muy familiarizados con la especie refiriéndose a ellos también como jabalís o cariblancos.

De las ocho cámaras utilizadas, dos de ellas se colocaron en fincas correspondientes al sector conocido como "Terrón" por ser una de las zonas más conservadas y por ser el parche más grande de bosque húmedo tropical maduro dentro del refugio (Ortiz-Malavasi, 2014). En noviembre del 2015, en una de ésta cámaras se registró una manada de chachos de monte de al menos ocho individuos (Fig. 1). En la fotografía se observan varios adultos y al menos dos juveniles.

\section{DISCUSIÓN}

El conocimiento ecológico local se define como un conjunto acumulativo de conocimientos, prácticas y creencias acerca de la relación de los seres vivos (incluido el ser humano) entre sí y con su medio ambiente (Grenier, 1999; Monroy-Vilchis et al., 2008). Este se obtiene mediante la convivencia e interacción prolongada con la fauna. Es importante utilizar el conocimiento ecológico local como complemento del conocimiento técnico y como guía para planificar y focalizar esfuerzos durante el desarrollo de investigaciones ecológicas, como en el caso del presente trabajo.

El RNVSM-CN está rodeado de agropaisajes por lo que se considera aislado de otras áreas protegidas (EscaleraReyes et al., 2013). Se ha demostrado que, en hábitats poco extensos, fragmentados y pocos diversos, los chanchos de monte son más susceptibles a desaparecer (Peres, 1996; Fragoso, 1999; Cullen et al., 2000; Fuller et al., 2002). Confirmar la presencia de chancho de monte es un hallazgo de gran importancia que puede servir para aumentar las medidas de conservación que se aplican en el refugio. Esta es una especie clave que se considera ingeniero ecosistémico (Beck et al., 2010; Altrichter et al., 2012). Al ser esta especie frugívora, influye en el reclutamiento y en la dispersión de semillas, principalmente de palma (Beck, 2006). Además, este hallazgo también puede ser útil para promover el estudio de los hábitats 
y agropaisajes colindantes, lo cual serviría para aumentar el conocimiento sobre las adaptaciones que presenta esta especie a hábitats que no son óptimos pero que permiten su supervivencia.

\section{AGRADECIMIENTO}

Agradecemos especialmente a A. Chuprine por su invaluable apoyo en ambas investigaciones. A J. Cortéz, V. Narváez, M. Aranda (ICOMVIS), P. Murillo, J. Zamora y a N. Torres (CIDE-DET-UNA) por su ayuda en la realización de las entrevistas. Al "Programa Jaguar" (ICOMVIS) por su apoyo en el financiamiento de una de las investigaciones. También se agradece a J. Sáenz por la facilitación de las cámaras trampa y a los funcionarios del RNVSM-CN por todo su apoyo durante el trabajo de campo.

\section{REFERENCIAS}

Alfaro, L. (2006). Estimación de la población de jaguares (Panthera onca) en el Parque Nacional Santa Rosa y Parque Nacional Guanacaste del área de Conservación Guanacaste, Costa Rica. Tesis de maestría, Universidad Nacional de Costa Rica, Heredia, Costa Rica.

Altrichter, M., Taber, A., Beck, H., Reyna-Hurtado, R., Lizarraga, L, Keuroghlian, A. \& Sanderson, E.W. (2012). Range-wide declines of a key Neotropical ecosystem architect, the Near Threatened white-lipped peccary Tayassu pecari. Oryx, 46(01), 87-98.

Amit, R. (2006). El jaguar (Panthera onca) en el sector San Cristóbal del Área de Conservación Guanacaste, Costa Rica: Densidad, abundancia de presas y depredación de ganado. Tesis de Maestría, Universidad Nacional de Costa Rica, Heredia, Costa Rica.

Amit, R. \& Fernández, G. (2012). Interacciones entre jaguar, puma y humanos en Guanacaste, Costa Rica. Informe Técnico. Programa Gente y Fauna, Asociación Confraternidad Guanacasteca. Guanacaste, Costa Rica.

Beck, H. (2006). A review of peccary-palm interactions and their ecological ramifications across the Neotropics. Journal of Mammalogy, 87(3), 519-530.

Beck, H., Thebpanya, P. \& Filiaggi, M. (2010). Do Neotropical peccary species (Tayassuidae) function as ecosystem engineers for anurans?. Journal of Tropical Ecology, 26(04), 407-414.

Bodmer, R., Aquino, R., Puertas, P., Reyes, C., Fang, T. \& Gottdenker, N. (1997). Manejo y uso sustentable de pecaries en la Amazonía peruana. Occasional paper of the UICN species survival comission 18. UICN-Sur, Quito, Ecuador.

Briceño-Méndez, M., Reyna-Hurtado, R., Calmé, S. \& García-Gil, G. (2014). Preferencias de hábitat y abundancia relativa de Tayassu pecari en un área con cacería en la región de Calakmul, Campeche, México. Revista Mexicana de Biodiversidad, 85(1), 242-250.

Cabrera, J., Protti, M., Urriola, M \& Cubero, R. (2003). Distribución y abundancia de Caiman crocodilus en el refugio Nacional de Vida silvestre Caño Negro, Costa Rica. Revista de Biología Tropical, 51(2), 571-578.

Carrillo, E., Wong, G. \& Sáenz, J.C. (2002). Mamíferos de Costa Rica. INBIO, Costa Rica.

Chávez, C., de la Torre, A., Bárcenas, H., Medellín, R.A., Zarza, H. \& Ceballos, G. (2013). Manual de fototrampeo para el estudio de fauna silvestre: El jaguar en México como estudio de caso. Alianza WWF-Telcel, UNAM, México.

Chinchilla, F. (1997). Dieta del jaguar (Panthera onca), el puma (Felis concolor) y el ocelote (Felis pardalis) Carnivora: Felidae, en el Parque Nacional Corcovado, Costa Rica. Revista de Biología Tropical, 45(3), 1223-1229.

Coloma, C., Caballero, L., Ricart, M \& Bueno, S. (2009). Diagnóstico del Refugio Nacional de Vida Silvestre Caño Negro ArenalHuetar Norte, Costa Rica. Universidad Autónoma de Barcelona. Barcelona, España.

Cullen, L., Bodmer, R.E. \& Padua, C.V. (2000). Effects of hunting in habitat fragments of the Atlantic forests, Brazil. Biological Conservation, 95, 49-56.

Escalera-Reyes, J., Feria, R.C. \& Aguilar, A.L.D. (2013). “Las apariencias engañan" Conservación, sociedad local y relaciones de poder: el caso de Caño Negro (Costa Rica). AIBR, Revista de Antropología Iberoamericana, 8(3), 369-394.

Fragoso, J.M.V. (1999). Perception of scale and resource partitioning by peccaries: behavioral causes and ecological implications. Journal of Mammalogy, 80, 993-1003.

Fuller, T., Carrilllo, E. \& Sáenz, J. (2002). Survival of protected White lipped peccaries in Costa Rica. Canadian Journal of Zoology, 80, 586-589.

Grenier, L. (1999). Conocimiento indígena: guía para el investigador. Editorial Tecnológica de Costa Rica y Centro Internacional de Investigaciones para el Desarrollo, Ottawa, Canadá.

Holdridge, L. (1979). Ecología basada en Zonas de Vida. Editorial IICA, Costa Rica.

Keuroghlian, A., Desbiez, A., Reyna-Hurtado, R., Altrichter, M., Beck, H., Taber, A. \& Fragoso, J.M.V. (2013). Tayassu pecari. En IUCN Red List of Threatened Species 2013: e.T41778A44051115. http://dx.doi.org/10.2305/IUCN. UK.2013-1.RLTS.T41778A44051115.en.

Leonardi, M. L., Amit, R., Watson, R., Gordillo, E. \& Carrillo, E. (2010). Presencia de Tayassu pecari (Atiodactyla: Tayassuidae) en Parque Nacional Rincón de la Vieja, Guanacaste, Costa Rica. Brenesia, 73/74, 146-147.

Mayer, J.J. y R.M. Wetzel. (1987). Tayassu pecari. Mammalian Species, 293, 1-7. 
Medellín, R.A., Azuara, D., Maffei, L., Zarza, H., Bárcenas, H., Cruz, E., Legaria, R., Lira, I., Ramos-Fernández, G., \& Ávila, S. (2006). Censos y monitoreos. Pp. 23-35 en Memorias del primer simposio "El jaguar mexicano en el siglo XXI: situación actual y manejo" (Chávez, C. y G. Ceballos, eds.) Conabio, Alianza WWF-Telcel y UNAM, México.

Monroy-Vilchis, O., Suárez, P., Zarco-González, M.M., RodríguezSoto \& Urios, V. (2008). Uso tradicional de reptiles, aves y mamíferos silvestres en la Sierra Nanchititla, México. UNAM, México.

Montalvo, V. (2012). Cambios en la abundancia, actividad temporal y dieta del jaguar (Panthera onca), otros felinos y sus presas en el Parque Nacional Santa Rosa, Costa Rica. Tesis de maestría, Universidad Nacional de Costa Rica, Heredia, Costa Rica.
Ortiz-Malavasi, E. (2014). Atlas Digital de Costa Rica. Tecnológico de Costa Rica, Cartago, Costa Rica.

Peres, C.A. (1996). Population status of white lipped Tayassu pecari and collared peccaries T. tajacu in hunted and unhunted Amazonian forests. Biological Conservation, 77,115-123.

Sowls, L. (1997). Javelinas and the other peccaries: their biology, management and use. Texas A and M University Press, College Station. Texas, EE.UU.

Wainwright, M. (2007). The mammals of Costa Rica: a natural history and field guide. Zona Tropical Publication, Cornell University Press, Nueva York, EE.UU. 
